\title{
La Metafísica de la mente de A. Kenny: 25 años después
}

\section{(A. Kenny's Metaphysics of Mind: 25 years later)}

\section{MIGUEL GARCÍA-VALDECASAS}

Universidad de Navarra, Pamplona

Instituto Cultura y Sociedad

garciaval@unav.es

Resumen. Coincidiendo con el 25 aniversario de la publicación de la Metafísica de la mente de A. Kenny, este artículo trata algunos de los principales puntos del libro. Concretamente, el dualismo de Descartes, la noción aristotélica de alma o psychê, el lenguaje humano y el animal, la acción voluntaria, la relación mente-cerebro, el pensamiento y la intencionalidad, y el determinismo y la libertad. El autor sostiene que, aunque el libro de Kenny contiene argumentos válidos inspirados en Wittgenstein y la tradición aristotélica, no siempre aprecia toda la profundidad de esta tradición en conceptos básicos como el de psychê o la inmaterialidad del entendimiento humano. A pesar de eso, el libro de Kenny es uno de los mejores esfuerzos hasta el presente por romper con Descartes y el empirismo británico,y por incorporar la tradición aristotélica a la filosofía de la mente contemporánea.

Palabras clave: filosofía de la mente; dualismo sustancial; Wittgenstein; Aristóteles.

Abstract. To mark the 25th anniversary of A. Kenny's The Metaphysics of Mind, this article discusses some of the central arguments of this book, in particular, it discusses Descartes' dualism, the notion of soul or Aristotle's psychê, human and animal language, voluntary action, the self, the mind-brain relation, thinking and intentionality, and determinism and free will. The author holds that, although Kenny's book offers valid and 
substantial arguments inspired in Wittgenstein's thought and the Aristotelian tradition, he occasionally fails to appreciate the depth of basic concepts in the Aristotelian tradition such as that of psychê and the immateriality of the human intellect. Despite this, the book constitutes one of the best efforts to break off with Descartes' and the empiricists' ideas, and to incorporate the Aristotelian tradition to the contemporary philosophy of mind.

Keywords: philosophy of mind; substance dualism; Wittgenstein; Aristotle.

The Metaphysics of Mind es una de las mejores aportaciones de A. Kenny a la filosofía analítica de la mente. Los editores del Festschrift que se publicó en 2010 dedicado a su pensamiento observan que «casi nunca se han reflejado ideas tan esclarecedoras y fecundas como las de este libro» (Hacker and Cottingham 2010, viii). Publicado en 1989 coincidiendo con el 40 aniversario de la publicación de The Concept of Mind, Kenny logró presentar en esta obra algunas de sus más destacadas ideas de obras anteriores. Sin haber tenido la difusión de la obra de Ryle por navegar contra corriente, The Metaphysics of Mind es igualmente ambiciosa. Ahora, a los 25 años de su publicación, me propongo examinar algunos de sus puntos nucleares.

Muchos consideraron la obra de Ryle un clásico y un punto de partida obligado en la filosofía de la mente. The Concept of Mind aborda los problemas centrales de la psicología filosófica, el más importante de los cuales es el problema alma-cuerpo, o en términos más precisos, la relación entre los estados mentales y físicos de un sujeto. En 1949, la filosofía del lenguaje se abría camino con la puesta en circulación de algunas ideas del último Wittgenstein que revelaban la importancia del lenguaje ordinario como instrumento de la especulación filosófica. Aunque Kenny declara que el lenguaje no es el único ni el más importante punto de partida del filósofo (Kenny 1989, viii), su obra tiene un marcado interés por el lenguaje. El autor se siente deudor de la filosofía de Wittgenstein, que marca gran parte de la orientación del libro. También destaca la capacidad de incorporar al presente conceptos de la tradición filosófica. Como es sabido, parte importante del bagaje de Kenny procede del aristotelismo. En su prólogo, aclara 
que «el sistema filosófico que trato de defender es una prolongación del aristotelismo medieval en el que recibí mis primeras enseñanzas en filosofía» (Kenny 1989, ix), por lo que muchas de sus ideas tienen la impronta metafísica de la tradición que representa Aristóteles, y su principal comentador medieval Tomás de Aquino.

El cuarto autor que es fuente de inspiración para Kenny es Descartes. El hilo principal de diversos capítulos es su crítica a la visión cartesiana del hombre y sus facultades. Para Kenny, el cartesianismo es una manera intuitiva de articular ideas que se introduce subrepticiamente en el pensamiento. Uno de sus principales opositores fue Wittgenstein, que se resistió a reconocer la existencia del cogito cartesiano y su principal vehículo: la introspección, esto es, la operación intelectual por la que un sujeto se autoconoce. Su reluctancia a aceptar la clave de bóveda del pensamiento cartesiano - el cogito-, marca el fin de la sustancia pensante o res cogitans, que para Wittgenstein es un mero sujeto de atributos mentales. En el caso de las emociones del alma, que Descartes agrupa bajo la categoría general de pensées, Kenny observa que el planteamiento cartesiano ha impregnado el pensamiento occidental hasta tal punto que muchos que se consideran sus detractores deben mucho a Descartes (Kenny 1989, 53).

El autor desea oponer a esta seductora e intuitiva visión de lo mental otra más coherente inspirada en el pensamiento de Wittgenstein y en la tradición aristotélica. Una de esas aportaciones es la noción aristotélica de psique, una noción que permite entender al ser humano como una unidad sustancial, y no, como proponía Descartes, como un agregado artificial de sustancias heterogéneas. Parte de su análisis consistirá en mostrar cómo se articula esa unidad en un ser humano que aparente poseer múltiples dimensiones.

A continuación, destacaré algunas de las ideas principales de la obra. No es mi objetivo analizar todos los capítulos del libro. Me centraré en algunos que tienen mayor relevancia en el contexto presente, y en otros que contienen aspectos polémicos, quizá por estar escasamente definidos por el autor, o por partir de una interpretación sesgada de la tradición aristotélica. En todos ellos, este análisis reconoce el mérito de los argumentos de Kenny. 


\section{El dualismo cartesiano}

Es sabido que la filosofía de Descartes establece una drástica separación entre alma y cuerpo. Esta separación incluye fenómenos que en la perspectiva de Descartes se originan en sustancias distintas. La mente, que es esencial en una de esas sustancias, la res cogitans, es parte central del alma; su principal operación es la conciencia. Para Descartes todo sujeto posee una capacidad de percepción interior que tiene un carácter único. Esta capacidad le permite conocer con infalibilidad los eventos que tienen lugar dentro de sí por introspección. Así, los fenómenos que ocurren en el alma tienen cualidades epistemológicas netamente distintas a las del cuerpo, porque mientras el sujeto percibe los fenómenos del alma y puede distinguir su origen, los fenómenos del cuerpo, por ser de diverso origen no son del todo fiables y por tanto, su grado de certeza es significativamente menor.

Así pues, según el dualismo conocemos nuestros propios estados de forma infalible y privada, y conocemos los estados mentales en que se hallan los demás hipotéticamente. Para Kenny, este esquema conduce a un escepticismo sobre los estados mentales de los demás y es engañoso sobre el modo en que conocemos los propios. Las objeciones al planteamiento dualista son varias. En primer término, es cuestionable que todo lo que sepamos acerca de las intenciones, deseos y emociones de los demás sean conjeturas inferidas de su comportamiento exterior. De ordinario, verbos psicológicos como «desear», «creer» 0 «tener la intención de» en su uso de tercera persona son mucho más que una descripción de los indicios que el comportamiento exterior de un sujeto nos da de lo que éste tiene en su mente. El significado de estos verbos va más allá. Entre otras razones, porque no los empleamos una vez que hemos observado su comportamiento, aunque de hecho lo hagamos así en ciertos casos. De ordinario sucede más bien al revés. Comprendemos las acciones de los demás toda vez que poseemos conceptos como «desear», «creer» y «tener la intención de» para darles sentido. A la luz de esos conceptos, nos hacemos cargo de sus deseos, creencias e intenciones en virtud de una pre-comprensión gramatical que en el caso de los seres humanos está estrechamente asociada a la obser- 
vación. Por tanto, no es que el que comportamiento de los demás nos dé pistas sobre sus intenciones, sino que las pistas que éste nos da son sólo comprensibles bajo la forma de intenciones. De hecho, a menudo las intenciones de los demás nos resultan más claras que sus efectos, es decir, que la identificación precisa de las consecuencias de sus acciones (Kenny 1989, 6).

Frente a dualistas - filósofos que como Descartes, convierten alma y cuerpo en dos sustancias - y conductistas - quienes acentúan la importancia de la conducta hasta eliminar los fenómenos del alma-, Wittgenstein adoptó un punto intermedio. Para él, los estados de la mente, ni son reductibles a sus expresiones, ni son completamente separables de éstas (Kenny 1989, 4). Para aclararlo, Wittgenstein estableció una distinción entre «síntomas» $\mathrm{y}$ «criterios», donde el síntoma tiene una relación inductiva con el fenómeno en cuestión, mientras que el criterio tiene una relación no-inductiva, o más bien lógica. Así, se puede decir que, mientras el dolor de cabeza puede ser un síntoma de gripe, el llanto amargo es un criterio o señal inequívoca de tristeza. No necesitamos inferir que un sujeto sufre o está sufriendo notablemente porque llora; lo comprendemos inequívocamente. Generalmente, mientras que los fenómenos naturales se conocen a través de síntomas, el comportamiento de los demás nos ofrece siempre un criterio. Aplicando esta distinción a la relación entre estados mentales y físicos, se puede decir que «ciertos estados o eventos del cerebro pueden ser síntoma de ciertos estados mentales, pero no pueden ser un criterio en el sentido en que lo es el comportamiento [de un individuo que está en un estado concreto]» (Kenny 1989, 5).

Por tanto, la idea cartesiana según la cual mis propios estados mentales son nítidos, mientras que los estados de los mentales de los demás se conocen hipotéticamente es falsa. El conocimiento de sí no es infalible. Con la caída del modelo infalibilista de conocimiento de sí cae también la noción cartesiana del yo. Para Descartes, la conciencia es la característica de lo mental por excelencia, el criterio que marca la diferencia entre hombres y animales. En cambio, para Kenny el criterio diferencial está en la capacidad de dominar los complejos símbolos de que está cargado el orden moral, social, científico, etc. (Kenny 1989, 7). Al situar la mente en la esfera de lo 
privado, Descartes la considera el lugar de estados psicológicos ocultos e inaccesibles a la observación pública. Sin embargo, «ni la comprensión aritmética ni el deseo de alcanzar fama son estados especialmente privados; el sujeto en cuestión no tiene una capacidad particular para pronunciarse sobre su presencia o ausencia» (Kenny 1989, 9). «Puedo creer que he entendido una operación aritmética concreta, pero tras ponerme a prueba mi profesor me puede mostrar que no. De forma análoga, quizá sólo un amigo pueda convencerme de que estoy iniciando una campaña política no por amor a la justicia, sino con el fin de salir en los periódicos» (Kenny 1989, 9).

La racionalidad tampoco es un fenómeno privado. El orden moral, social y científico de la vida humana tiene un indudable carácter social y público. Por eso, la capacidad de dominar lenguajes distintos a través de múltiples contextos pone en evidencia la validez del criterio subjetivo de conciencia como el rasgo más destacado de lo psicológico.

Cuando la noción cartesiana de conciencia parecía abandonada, Kenny señala que algunos filósofos contemporáneos de la mente la han recuperado. La noción de sensory qualia como un fenómeno interno de carácter inexpresable es buena muestra de ello. La noción de qualia tiene una larga y variada historia en la filosofía analítica (Crane 2000), con significados variados para autores distintos. En los últimos decenios el término ha venido siempre asociado a fenómenos de carácter interno e inefable cuyo conocimiento por parte de los demás es solamente conjetural, y en algunos casos imposible. Algunos han concebido el dolor, p. ej., como una cualidad intrínseca (qualia) que sólo resulta accesible al sujeto por introspección. En algunas ocasiones los qualia han derivado en givens, que según sus defensores son objetos de carácter interno cuya traducción en conceptos o su equiparación con ellos entraña su desnaturalización. Para sus defensores, si se clasifica a estos objetos bajo la etiqueta de «sensaciones» perderían inmediatamente su carácter de givens, que consiste precisamente en resistir toda conceptualización. De esta forma, con la aparición de givens en teorías fundacionistas, la filosofía contemporánea se ha vuelto a sumergir en esquemas que reducen ciertos ámbitos de lo mental a la esfera cartesiana de los fenómenos privados. 


\section{El alma, el lenguaje y la intelección animal}

Como consecuencia de la separación de alma y cuerpo en la filosofía cartesiana, se podría pensar que los seres humanos «tenemos» cuerpo. Y así es, pero tener un cuerpo no es incompatible con «ser» cuerpo, porque no somos «algo que está sobre y por encima del cuerpo» (Kenny 1989, 17). De acuerdo con una idea íntimamente aristotélica, Kenny considera que no somos algo distinto a nuestro cuerpo, algo que «tiene» un cuerpo que le pertenece en el modo como una casa pertenece a su dueño. «Somos» fundamentalmente un cuerpo y un conjunto de facultades entre las que destaca el entendimiento y la voluntad. Pero así como es evidente que somos seres corporales, por las connotaciones religiosas que tiene el término «alma», vinculadas a la existencia de un alma inmortal en los seres dotados de entendimiento y voluntad, puede parecer menos evidente que tenemos un alma. Por eso, Kenny advierte que debemos entender cuidadosamente este término. «Los filósofos de la tradición aristotélica emplearon los términos griego y latino que se corresponden con la palabra «alma» no para significar algo más que el intelecto, sino para significar algo menos» (Kenny 1989, 18). Para los griegos, el alma es un principio de vida, pero este principio no es necesariamente inmortal. Si en la tradición aristotélica, el alma humana resulta ser inmaterial e inmortal, lo es por algo propio de los seres humanos, no por algo propio de su principio de vida. La vida de plantas y animales se acaba con su muerte. En este sentido, el autor añade que el alma o principio de vida aristotélico, en sus niveles inferiores, no tendría por qué ser «necesariamente inmaterial», ya que la psique de una planta podría coincidir prácticamente con su ADN (Kenny 1989, 18).

Evidentemente, afirmar que la psique de una planta no es «necesariamente inmaterial» en la tradición aristotélica es precipitado, y no pasaría el escrutinio de la mayor parte de la opinión crítica. El ADN de una planta y el de cualquier otro animal no es sólo materia orgánica, sino sobre todo, el conjunto de instrucciones necesarias para la generación de proteínas. El ADN es así «materia informada». Sus instrucciones no vienen dadas por el hecho de estar compuestas de material genético, pues uno podría fa- 
bricar otros instrumentos de material genético que no desempeñen las funciones del ADN en los seres vivos. Las instrucciones vienen definidas por el hecho de que ese material está informado y cumple una función en el conjunto del viviente. El ADN, por tanto, tiene un carácter formal y como materia informada debe exceder las capacidades de la materia de la que está compuesta el material genético. Todo lo cual es bien sabido por Kenny, quien tal vez admitiría la especificidad de las dimensiones formales de lo vivo, pues el resto de capítulos es coherente con esta idea.

El sentido más común de la palabra «alma» es específico del ser humano. Aunque los animales tengan psique, solo de los seres humanos se dice que tienen propiamente un alma (Kenny 1989, 19). Tras lo visto anteriormente, ya no es necesario decir que el alma se conoce por introspección, es decir, observando atentamente la aparición de fenómenos interiores. Esta idea no se sostiene coherentemente con la crítica a la introspección cartesiana. Para Kenny, «la mente humana es la capacidad de adquirir habilidades intelectuales (...) Es una capacidad de segundo orden: una habilidad de adquirir o poseer habilidades» (Kenny 1989, 20). Entre ellas, «la habilidad más importante es dominar un lenguaje (...) El estudio de la adquisición y el ejercicio del lenguaje es el medio por excelencia que permite estudiar la naturaleza de la mente humana» (Kenny 1989, 20). A diferencia de los sentidos, que son capacidades innatas que se activan nada más nacer, o en el transcurso de los primeros días o semanas, el lenguaje se adquiere con el tiempo. Pero con él, llegan nuevas capacidades. Para Kenny, sólo es posible adquirir habilidades como las matemáticas, y tras ella, otras habilidades numéricas, una vez que se ha aprendido un lenguaje. De ahí que el lenguaje actúe como vehículo de la adquisición de nuevas habilidades, incrementando sucesivamente la capacidad de lograr nuevas habilidades sobre la base de las ya conseguidas. Se podría decir así que el lenguaje es la llave que abre la puerta al resto de habilidades, o una habilidad de habilidades.

La afirmación de que el lenguaje es la capacidad humana por excelencia podría sorprender. En la tradición aristotélico-tomista, no hay una capacidad central sino dos: entendimiento y voluntad, pero ninguna de las dos funciona adecuada y sostenidamente sin el resto de facultades humanas, 
tanto apetitivas como sensitivas. La mayor parte de ellas son orgánicas. Aunque Aristóteles advirtió que el lenguaje es una característica exclusivamente humana, que los animales tienen voz y pueden comunicarse mutuamente la sensación de dolor y placer, y que el ser humano es el único que tiene palabra (logos) (Aristóteles 2000b, 1253a), en la tradición aristotélica el lenguaje no es parte de las capacidades centrales del hombre.

Lo que Kenny, siguiendo a Wittgenstein, entiende por «lenguaje» procede de la tradición analítica. En esta tradición, el lenguaje, entendido en un sentido más amplio que la mera capacidad expresiva o comunicativa, es el núcleo duro de la racionalidad. El último Wittgenstein, en concreto, tiene una peculiar concepción de la «gramática» como la base primordial del lenguaje. Wittgenstein llama «gramática» al conjunto de reglas que subyacen a ciencias y prácticas humanas como las reglas aritméticas, las normas morales o los cánones artísticos, por citar sólo algunos campos en los que las reglas saltan a la vista. La comprensión y el dominio de los conceptos sobre los que se fundamentan, así como su correcta aplicación, excede el simple manejo de un vocabulario. Veamos porqué. En un vocabulario, un término está simplemente por un significado. Pero un sujeto que aprende aritmética no solamente aprende a emplear correctamente el vocabulario de los números; también aprende otras capacidades que manifiestan la comprensión del contexto en el que pueden o deben emplearse. P. ej., una adecuada comprensión de una serie numérica lleva consigo el conocimiento de que en la serie 1, 3, 5, 7, el siguiente número es el 9, y el siguiente el 11. Sólo cuando esto se entiende, se ha entendido la serie. Para continuarla, un sujeto que demuestre haber comprendido las «normas» de la serie debe aprender a determinar el siguiente número. Como toda gramática establece reglas de uso, Wittgenstein identifica el comportamiento conforme a reglas como la parte esencial de los elementos de un lenguaje.

Esta concepción rebasa los límites tradicionales del concepto de lenguaje. Para Kenny, siguiendo a Wittgenstein, tener un lenguaje es más que el fenómeno psicológico de pensar. Kenny observa que el lenguaje diferencia a los hombres de los animales. Aunque los animales tienen experiencia del dolor y lo sienten, carecen del concepto de «dolor». Los humanos 
compartimos con los animales la experiencia del dolor, pero una vez que aprendemos el lenguaje adquirimos su concepto, y, siguiendo a Wittgenstein, la forma adecuada de emplearlo. Ahora bien, más adelante reconsidera su posición y estima que no hay nada antropomórfico en atribuir conceptos a los animales (Kenny 1989, 36), a quienes se les puede atribuir únicamente conceptos que resultan expresables de forma no verbal, es decir, a través de su comportamiento. Cuando un animal manifiesta la intención de salir de casa, se le puede atribuir lo que revele su comportamiento. Pero aunque Kenny no lo mencione explícitamente, existe una gran diferencia entre disponer o no del concepto de dolor. Al aprender su significado y su expresión, los seres humanos aprendemos a transformar, y en cierto modo trascender, la experiencia del dolor, pasando de una sensación a una caracterización racional de este tipo de experiencia. Con ella se puede explicar la relación entre el dolor y el sufrimiento. Sólo la comprensión del dolor en clave lingüística pudo llevar a C. S. Lewis a escribir A Grief Observed, un libro que describe y articula magistralmente los efectos del sufrimiento en primera persona, traspasando los límites del sufrimiento. Esto prueba que el dolor expresable no verbalmente y el expresable lingüísticamente distan mucho entre sí.

Kenny anuncia una verdad empírica: la capacidad de pensar es característica de los seres humanos (Kenny 1989, 24). Sin embargo, más adelante se retracta, y observa que la capacidad de pensar no es exclusiva de ellos (Kenny 1989, 127). Los animales también piensan, aunque su pensamiento no sea fruto del entendimiento (Kenny 1989, 24), o de una capacidad comparable. Su actividad se limita a la posesión de «pensamientos simples». Así pues, hemos de suponer que un animal puede «pensar que $p$ ». Pero al no «pensar que $p$ » mediante conceptos, su «comprensión» saca a relucir limitaciones evidentes. Una de ellas es la falta de reflexividad. Kenny llama reflexividad a la capacidad del pensamiento humano de «pensar que se piensa que $p$ ». Así, un animal podría «pensar que $p »$, pero no «pensar que piensa que $p »$; nada en su repertorio conductual nos induce a creer que aprecian la diferencia entre ambas expresiones (Kenny 1989, 23, 39); de lo contrario su conducta sería muy distinta. En cambio, la reflexividad 
permite identificar razones para actuar y articularlas en descripciones lingüísticas como «hice $\mathrm{X}$ porque $\mathrm{Y}$ ». La falta de reflexividad impide que los animales conozcan sus fines como fines, y así, que logren articular las razones que les mueven. Así pues, aunque los animales tengan «pensamientos simples», parece evidente que no actúan propiamente por razones.

Esta idea ha provocado cierto debate. MacIntyre (MacIntyre 1999, 54), leyendo a Tomás de Aquino, reconoce a los animales una participación en la virtud de la prudencia, la llamada «prudencia natural», que les da cierta capacidad de actuar con arreglo a ciertos «juicios naturales». MacIntyre admite, siguiendo también a Tomás de Aquino, que la capacidad de emitir y evaluar juicios es exclusivamente humana. Pero piensa que la diferencia entre la mente animal y la humana no está bien recogida cuando se mantiene que sólo los seres humanos son capaces de lenguaje. La cuestión no está en la capacidad de articular «expresiones semánticamente significativas» de la que algunos animales parecen ser capaces. Lo que caracteriza la mente humana es la consideración y evaluación de razones para actuar como buenas o malas, mejores o peores. Así, aunque deba acentuarse la diferencia existente entre las razones de los animales y las de los seres humanos, no hay porqué negar que los animales tienen «razones para actuar». De hecho, los seres humanos compartimos razones para actuar con los animales que son anteriores a cualquier reflexión, y que están básicamente presentes en el instinto. Precisamente, la racionalidad se cifra en la capacidad, que un niño adquiere con su desarrollo, de identificar nuevas o mejores razones a las que posee antes de cualquier reflexión. Así pues, el debate sobre la racionalidad animal sigue abierto, y no parece que Kenny lo haya resuelto con su resistencia a conceder una capacidad lingüística a los animales.

Otros filósofos contemporáneos como Glock (Glock 2009) han cuestionado la idea de que los animales no puedan actuar por razones. Para Glock, si «actuar por razones» significa que el comportamiento del animal responde a una determinada descripción lingüística, él no ve obstáculos para decir que los animales obran «intencionalmente», puesto que su comportamiento parece estar guiado por intenciones descriptibles lingüísticamente. Lo 
prueba p. ej. su capacidad de comprender hechos objetivos y deliberar para satisfacer sus deseos.

\section{Acción voluntaria y libertad}

Su teoría de la voluntad tiene la peculiaridad de asignar a los animales un tipo de deseos (wants) específicos, distintos de las meras necesidades (needs) de la vida vegetal. Kenny acepta la definición tradicional de los deseos como necesidades sentidas. Para tener deseos de este tipo estima que es imprescindible ser un agente capaz de obrar. Obrar es algo que, según el autor, también seres inanimados pueden hacer; se trata de un comportamiento «natural» que proviene de una potencia natural, como lo es la potencia expansiva de un gas en un recipiente. Pero los agentes animados, a diferencia de los inanimados, deben tener a su disposición un repertorio más o menos variado de formas de conseguir fines. «El agente debe tener todo un repertorio de diversas acciones que permitan conseguirlos, no una única forma característica. Y no sólo eso, sino que debe poner en práctica dichas alternativas en situaciones en las que no media la necesidad. Tanto animales como plantas se alimentan, pero sólo los animales juegan» (Kenny 1989,34$)$. La característica esencial, en definitiva, es que los deseos sólo pueden atribuirse a seres conscientes de sus necesidades (Kenny 1989, 34). Por supuesto, los seres humanos, que comparten con los animales parte de su naturaleza, están incluidos aquí. Por tanto, hasta ahora tenemos un tipo de conducta propia de seres inertes, y otra propia de seres vivos.

Entre los seres vivos destaca el obrar humano. Éste se basa en la diferencia existente entre los deseos animales y los deseos humanos (Kenny 1989, 35). Para expresarla correctamente, Kenny designa cada tipo de conducta con términos distintos. Llama al deseo de los seres humanos «volición» (volition), y al animal, lo que podríamos llamar, para distinguirlo de la otra clase de «deseo» (want), «simple deseo» (pure desire). Una diferencia importante entre el simple deseo y la volición es que el primero demanda una satisfacción inmediata, mientras que la volición se extiende indefinidamente en el tiempo, y no tiene por qué estar presente constantemente 
en el flujo de la conciencia (Kenny 1989, 36). La volición lleva consigo el uso de conceptos que demandan una expresión lingüística, como se ha señalado antes en el caso de los conceptos humanos, mientras que los deseos se pueden expresar extralingüísticamente. Con todo, Kenny insiste en que las voliciones humanas son esencial o constitutivamente deseos (wants). Para entenderlo, podría precisarse que es como si el lenguaje sobreviniera sobre un tipo más básico de inclinación natural compartida por animales y seres racionales.

El autor destaca que a algunos filósofos les parece un antropomorfismo defender que los animales son capaces de acciones voluntarias. «En algunas discusiones sobre la libertad (free will) los filósofos parten de la suposición de que los animales son incapaces de acciones voluntarias» (Kenny 1989, 37). Para Kenny, en cambio, no hay nada que impida atribuirles un querer específico. La clave está en la definición de una acción voluntaria. «Si una acción voluntaria se define por el hecho de quererse (y no porque uno se vea forzado u obligado a hacer lo que no desea), cuando los animales obran para satisfacer sus deseos obran voluntariamente» (Kenny 1989, 37). P. ej., el perro que se inquieta al ver a su dueño descorrer el pestillo de la puerta «quiere» salir de casa. Como MacIntyre (MacIntyre 1999, 25) ha puesto igualmente de manifiesto resumiendo algunos estudios de campo realizados con delfines, estos animales logran resolver situaciones complejas mediante el desarrollo de diversas estrategias adaptadas a la reacción de su presa. Si dichas estrategias dan a los delfines una razón para actuar, parece que no actúan bajo la «obligación» del instinto, sino que, conocidas las alternativas, eligen el mejor curso de acción. Si las acciones voluntarias se definen, según la opinión de algunos filósofos, como acciones que se ejecutan en circunstancias donde siempre podría obrarse de otro modo, entonces parece evidente que los animales con un cerebro suficientemente desarrollado como es el caso de los delfines pueden escoger entre alternativas y obran por querer. Para Kenny, un animal puede obedecer a la voz de su amo o seguir otra inclinación (Kenny 1989, 38), por lo que mantener que no está forzado a tomar ninguno de los dos cursos de acción no es un antropomorfismo. 
Que los animales actúen voluntariamente no significa que sean libres. Únicamente significa que nada les hace incapaces de querer las acciones que desean. La libertad es un obrar racional, y su característica no es querer, sino el obrar intencionalmente. Para Kenny, sólo los seres humanos obran intencionalmente, o lo que es lo mismo, con arreglo a razones. Por «acción intencional» no nos referimos sólo al hacer X para obtener Y, es decir, a la persecución sostenida de fines. «Para mostrarlo es suficiente con observar que incluso agentes no conscientes como las plantas y los órganos de los animales, actúan para producir determinados estados de cosas. Las raíces de las plantas tienen [minúsculos] pelos para atraer hacia sí la humedad; sin embargo, ni las raíces ni sus [minúsculos] pelos albergan intenciones con respecto a la humedad» (Kenny 1989, 38). Evidentemente, a diferencia de una planta un animal alberga intenciones. Además, algunos animales añaden a este tipo básico de obrar la conciencia de esos fines. Pero a pesar de que los animales pueden hacer X para lograr Y, no pueden aducir ni articular las razones del porqué. No sólo porque no puedan hablar, sino porque Para Kenny carecen de la capacidad de comprender razones. Solamente los seres que son capaces de comprender los motivos de sus actos pueden actuar por razones. En consecuencia, para actuar intencionalmente no basta obrar por fines ni ser consciente de ellos, sino que es preciso hacerlo por razones y saber explicarlas (Kenny 1989, 39).

En la tradición aristotélica, la afirmación de que un animal obra por «querer» podría desconcertar al lector, con lo que sería precisa alguna aclaración terminológica. Así expuesta, la tesis de Kenny comporta que los animales actúan voluntariamente. Pero aunque pueda sorprender, esta forma de entender la conducta animal es aristotélica. Aunque es sabido que Aristóteles no tenía un concepto específico de voluntad, estudió con notable interés la acción humana. Distinguió la acción que se hace por ignorancia o coacción, de aquella que se hace espontáneamente, sin esas limitaciones. Para Aristóteles, toda acción que tiene por principio el deseo (orexis) puede considerarse una acción «voluntaria», en el sentido de que está guiada por un tipo de deseo. En ese sentido, es aristotélico decir que los animales obran voluntariamente (Aristóteles 2000a, 1111b9) en cuanto 
que sus acciones tienen origen en un deseo. Al seguir sus deseos, un animal obra por un principio consciente, y no por «resortes mecánicos», tal y como pensó Descartes. No obstante, para Aristóteles la voluntariedad animal es incompleta. La voluntariedad completa o perfecta es la voluntariedad humana, que Aristóteles llama proaíresis, y que se caracteriza por ser un deseo deliberado (Aristóteles 2000a, 1113a 10).

$\mathrm{Al}$ emplear el término «querer» para explicar el origen de las acciones voluntarias, Kenny no pretende dar a entender que los animales participan de la proaíresis aristotélica. Su repetida insistencia en que sólo aquel agente que es capaz de dar razones de sus actos puede obrar intencionalmente acerca su posición a la de Santo Tomás. Éste definió la acción voluntaria como una acción con conocimiento del fin y de la proporción de los medios con respecto al fin (Tomás de Aquino 1950-1956, I-II, q. 6. a. 2 c). Es decir, debe conocerse no sólo la relación medio-fin, sino la naturaleza del medio en cuento medio, y del fin en cuanto fin. Sin el conocimiento de esa «proporción» no se podrían albergar intenciones. Aunque Kenny atribuya a los animales conceptos y «pensamientos simples», al carecer de un lenguaje éstos son ineficaces para proporcionar las razones que les mueven. Por eso, aunque la admisión de un querer específico en los animales pueda resultar a priori contraintuitiva, su postura coincide en muchos aspectos con la de Santo Tomás. Sean o no conscientes de sus deseos, los animales no tienen el concepto de fin ni de las variadas formas que pueden adoptar.

\section{El yo de los filósofos}

El capítulo sobre el yo es, de nuevo, un ataque a una de las versiones menos conocidas del dualismo. Uno de los riesgos derivados del pensamiento cartesiano es entender el yo como una sustancia homogénea y persistente que permanece dentro de nosotros en un lugar oculto. El yo de Descartes, que Kenny llama también «el yo de los filósofos» por su asombrosa capacidad de atraer a los filósofos que han seguido a Descartes hasta nuestros días, se ve continuamente afectado por numerosas sensaciones externas e internas. Estas le ponen a la vista experiencias de los que el sujeto es in- 
faliblemente consciente. La crítica a esta idea que sigue a continuación se inspira en las críticas de Wittgenstein a las diversas doctrinas metafísicas del yo que tienen como origen la res cogitans, esto es, una supuesta sustancia metafísica a la que están referidos los usos del pronombre personal de primera persona.

«El propósito de este capítulo es mostrar que el yo de los filósofos es un mito, así como el yo de los poetas y dramaturgos» (Kenny 1989, 87). Este yo es una entidad sin otros rasgos que la autoconciencia y la propia creencia de que uno es «eso» a lo que se refiere el pronombre personal. La idea, conectada con la privacidad de los actos internos que es propia del dualismo cartesiano, ya nos resulta familiar. En sus Meditaciones Metafísicas Descartes señala que, aunque eventualmente pudiera dudar de su cuerpo, ¿cómo podría dudar de su existencia? Y se pregunta: ¿qué sería, en este caso, eso que duda? (Kenny 1989, 89). Una y otra vez, la pregunta por el objeto de la duda desemboca en un yo consciente. Descartes habla de él como de un poderoso foco de vida consciente que consuela a quien se ve acosado por la duda metódica. Pero este yo no es - mantiene Kenny- personal, o sea, el yo de una persona de carne y hueso, sino que es, por decirlo así, un punto consciente al amparo de toda duda. ¿Pero es éste un ser de carne y hueso? Para Kenny, es sólo un producto de la imaginación de Descartes. «Cuando Descartes se identifica a sí mismo con los contenidos de su mente, con lo que realmente se identifica es con los contenidos de su imaginación, no de su mente» (Kenny 1989, 90). Los escolásticos habrían identificado esta visión con los «fantasmas» de la imaginación creativa, que no tienen más entidad que aquella que le prestan las facultades interiores.

A la vez, Descartes, Locke y todo filósofo que hable del yo como de una entidad de la que pueden predicarse atributos directamente habría caído en el sugerente y malicioso error de confundir una parte de la sustancia con el todo, tomando así lo más evidente -el yo consciente- por la esencia del ser humano. Esta confusión, que en otra de sus obras llama «la falacia del homúnculo» genera un problema no pequeño de atribución lingüística que fue correctamente identificado por Aristóteles. En un célebre pasaje de De Anima, Aristóteles escribió que: «afirmar (...) que es el alma quien 
se irrita, sería algo así como afirmar que es el alma la que teje o edifica. Mejor sería, en realidad, no decir que es el alma quien se compadece, aprende o discurre, sino el hombre en virtud del alma» (Aristóteles 1978, 408b 12-15).

Quizá se piense que, al minar la sustancialidad del yo, Kenny suscribe el planteamiento escéptico. No es así; entre otros motivos porque la perspectiva de Hume (Kenny 1989, 90-92) hace inabordable la consideración del sujeto como problema filosófico. De la misma forma en que el ojo no puede verse a sí mismo, tampoco el sujeto de los estados psicológicos de Hume logra concebirse a sí mismo. Al descartar una solución posible del problema, el escéptico se limita a constatar la inexistencia del problema del yo. Pero para Kenny, es un problema real.

El yo de Descartes y los empiristas es un mito, un «ejemplo del sinsentido filosófico» (Kenny 1989, 87) y una curiosa entidad metafísica creada por una malinterpretación del pronombre reflexivo. El «yo» no se refiere a la persona que lo emplea de la forma en que un nombre se refiere a su portador (Kenny 1989, 88). Para explicarlo, Kenny introduce una sutil distinción entre el «yo» (my self) y «mi-yo» (myself). El yo es el foco o punto de vida consciente que comparece en los actos conscientes. Mi-yo, en cambio, va mucho más allá. Así, una cosa es creer que quien escribe estas líneas es quien escribe estas líneas, y otra que el «yo» es simplemente la persona que escribe estas líneas («mi-yo»). Mi-yo es el ser humano de cada cual integrando todos sus aspectos, no sólo aquellos de los que uno es consciente, sino también aquellos de los que podría no serlo y tal vez los demás sí. Y sabemos por qué; anteriormente se ha señalado que el cogito cartesiano es una quimera y autoconocimiento es falible. Así, mi-yo no está dado a la conciencia subjetiva. Lo que se puede saber de él está a la vista. Los demás pueden conocerlo tan bien o mejor que yo prestando atención a mis expresiones, mis gestos y, sobre todo, a mi modo de comportarme. Así, mi-yo es más complejo y notablemente polimórfico. Comprende la mente, la imaginación, las emociones, el cuerpo y sus estados, etc., todos los cuales entran dentro del sujeto que se es en la misma medida que los actos de conciencia. 


\section{La relación entre mente y cerebro}

En el capítulo sobre el intelecto, Kenny desarrolla algunas ideas dispersas por los cap. 2 y 5 que tratan respectivamente sobre el compuesto humano, y sobre las capacidades, facultades, potencias y disposiciones de la mente.

En el cap. 5, Kenny traza los sentidos principales del término «capacidad», «poder» $\mathrm{y}$ «oportunidad». Mientras que el poder es algo interno al sujeto, la oportunidad, como una circunstancia que permite el desarrollo de una capacidad, es algo externo a ella, y -se podría añadir-contingente; las oportunidades se dan o no se dan; las capacidades son, de ordinario, estables. El sentido más interesante del término «poder» para las facultades de la mente es el poder como «capacidad». La capacidades humanas de nadar, dibujar o hacer una división contrastan con las capacidades naturales, como p. ej., la capacidad del agua de mojar. La diferencia está en que, dada una oportunidad, las capacidades humanas pueden activarse o no, mientras que, en las mismas condiciones, las capacidades naturales se activan necesariamente.

Kenny identifica una distinción fundamental entre la capacidad, su posesor y su vehículo (Kenny 1989, 71). El posesor de una capacidad es quien la ostenta. Su posesor es una sustancia en sentido completo. Si un sujeto cree que $p$, esta creencia es suya, y no de su mente o de su capacidad lingüística. Sólo el sujeto puede ser el foco de atribución de capacidades. A este respecto, resulta ilustrativa la conocida afirmación de Wittgenstein: «sólo de un ser humano y de lo que se comporta como un ser humano vivo se puede decir: tiene sensaciones; ve, está ciego; escucha, está sordo; es consciente o inconsciente» (Wittgenstein 1953, §281). Evidentemente, en la jerarquía de capacidades de un ser humano, también caben distinciones conceptuales que permiten atribuir a determinadas facultades al ejercicio de una capacidad. La memoria, p. ej., es la capacidad de retener ideas en la mente tras su consideración. Así, aunque se podría decir que la memoria «recuerda», quien lo hace en realidad es el sujeto. Kenny señala que «el materialista está en lo cierto al decir que describir la mente es describir, en un cierto nivel de abstracción, un objeto físico. Pero el objeto físico que 
describen los predicados psicológicos es el ser humano, no su cerebro» (Kenny 1989, 151).

El vehículo es el medio físico por el que se ostenta una capacidad. «Un vehículo es algo concreto, que puede ser pesado y medido. Una capacidad, en cambio, no tiene ni longitud, ni altura ni ubicación» (Kenny 1989, 72). En el cap. 2, señala que una capacidad es algo que no necesariamente ha de ocupar un espacio (Kenny 1989, 27). «Mi mente no está en un espacio excepto en el sentido de que está allí donde está mi cuerpo» (Kenny 1989, 28). Esto no significa, lógicamente, que la mente está contenida dentro del cuerpo, que está unida a él por alguna de sus partes o distribuida entre ellas. Por esa razón, expresa que «la mente no es una sustancia física» (Kenny 1989, 74) incluso aunque el cerebro pueda ser el vehículo de esta capacidad. En ese sentido, Kenny advierte una distinción real entre mente y cerebro: «hay una distinción entre la estructura de la mente (...) y la estructura del cerebro como su vehículo» (Kenny 1989, 75).

Como consecuencia, la identificación entre mente y cerebro es «completamente desorientada» (Kenny 1989, 30). Uno de los errores más comunes en la filosofía contemporánea de la mente es identificar la mente y su vehículo. En un ensayo posterior (Kenny 2008) Kenny bautiza este reduccionismo como el «reduccionismo del vehículo», y consiste en creer que la mente está físicamente contenida en el cerebro o que es igual a él. El reduccionismo del vehículo ignora una verdad esencial, a saber, la contingencia de la relación mente-cerebro. Para Kenny, la relación mente-cerebro expresa una verdad empírica, que conocemos por observación y análisis científico. Pero la ciencia, a diferencia del análisis del lenguaje y su «gramática» es un saber a posteriori. Mientras que el análisis filosófico del significado de los verbos psicológicos, que nos habla de la relación entre mente y comportamiento, arroja conexiones necesarias, el análisis de los verbos psicológicos no nos dice nada sobre el cerebro. De donde surge una conclusión importante, aunque meramente probabilística según Kenny: así como puede haber cerebros sin mentes, como p. ej., cerebros que hayan pasado su vida en una probeta, también es concebible la existencia de mentes sin cerebro (Kenny 1989, 30). Años más tarde, en Cognitive Scientism dirá 
que «no hay nada incoherente en la idea de que una capacidad logre existir sin un vehículo material» (Kenny 2008, 162), aunque está por ver que ese sea el caso de la mente. Lo que acepta como posibilidad es la existencia de capacidades sin vehículos.

En ese mismo ensayo, cita un importante texto de Wittgenstein: «ninguna suposición me parece más natural que la de que no haya un proceso cerebral correlativo o asociado con el pensamiento (...) Es perfectamente posible que ciertos fenómenos psicológicos no puedan ser investigados fisiológicamente, porque fisiológicamente nada se corresponda con ellos. Vi a este hombre hace años: lo reconozco, recuerdo su nombre. ¿Por qué tiene que haber una causa de su recuerdo en mi sistema nervioso? ¿Por qué no puede existir una regularidad psicológica que no se corresponda con una regularidad física? Si esto trastoca nuestro concepto de causalidad, bienvenido sea» (Wittgenstein 1974, §§608-610).

Wittgenstein parece dejar abierta la posibilidad de que los estados de la mente no se correspondan con los del cerebro por una causalidad estudiable científicamente, esto es, mediante regularidades que tengan carácter de ley. Sin embargo, no todos entienden que esta postura es incompatible con el materialismo. Es sabido que Davidson contempla la posibilidad de una relación anómala entre los estados mentales y cerebrales. Para Davidson la identidad entre estados mentales y cerebrales no lleva necesariamente consigo una conexión estudiable mediante leyes. Aunque los estados mentales y cerebrales no sean respondan a leyes, y de esta forma no se puedan identificar estados genéricos de la mente con estados genéricos del cerebro con carácter universal, Davidson postula que se identifican caso a caso en una relación de uno a uno. Así pues, el materialismo sería posible a pesar de la falta de una correlación nomológica entre los estados mentales y cerebrales.

Ciertamente, el llamado «monismo anómalo» de Davidson (Davidson 1980) ha caído en descrédito por su dificultad para ser entendido, así como por la falta de argumentos de fondo que lo justifiquen.

Kenny admite poseer sentimientos encontrados ante el pasaje de Wittgenstein. Por una parte, el pasaje de Wittgenstein subraya la contingencia 
de la relación mente-cerebro, lo que hace inasumible el reduccionismo del vehículo y la defensa de un planteamiento materialista. Por otra, Wittgenstein despierta la posibilidad de que el cerebro no sea el órgano o el vehículo de la mente. Pero ésta no es su postura en The Metaphysics of Mind, una obra en la que el autor se compromete con la idea de que toda capacidad, incluida la capacidad de razonar, tiene un vehículo físico. Señala: «el vehículo de la mente humana es, muy probablemente, el cerebro humano» (Kenny 1989, 73). Esta probabilidad no está vinculada a la posibilidad de que la mente carezca de un vehículo, sino a la contingencia de la relación mente-cerebro.

El pasaje de Wittgenstein se podría interpretar como un avance estratégico para defender una tesis aristotélica: la inmaterialidad del intelecto. Kenny señala que Wittgenstein concibe «la posibilidad de un alma aristotélica o entelecheia que opera sin vehículo material, una causa formal o final a la que no corresponde ninguna causa eficiente mecánica» (Kenny 2008, 162).

Es sabido que años atrás, en Aquinas on Mind, estudió y rechazó los argumentos de Tomás de Aquino para probar la inmaterialidad del intelecto. Como es sabido, la Summa Theologiae propone la inmaterialidad del conocimiento a partir de la posibilidad real de concebir formas puras como la forma de una piedra. El argumento sigue estas líneas: si el entendimiento estuviera compuesto de materia, nunca llegaría a conocer formas puras, esto es, las formas de las cosas sin materia, sino las formas afectadas de materialidad como son las formas individuadas de las cosas. «Si el entendimiento estuviera compuesto de materia y forma», dice Tomás de Aquino, «sólo podría conocer las formas individuales, tal y como es el caso de los sentidos, que reciben las formas de las cosas mediante un órgano» (Tomás de Aquino 1950-1956, I, q. 75, a. 5, c).

Kenny no pone reparos a la idea de que el entendimiento tenga como objetos de conocimiento «formas puras» o conceptos universales. Tampoco pone reparos a la identidad efectiva entre el cognoscente y lo conocido en el acto de conocer. Su crítica se dirige más bien al modo como se presenta el argumento de Tomás de Aquino. El punto en cuestión es la solución al 
problema de la atribución de un concepto. Kenny acepta que la idea de una pirámide es la idea de pirámide, y no la idea de una pirámide concreta. Pero ¿qué hace que la captación de un concepto sea la captación de ese concepto? Un concepto no se individúa por lo que cae bajo él -lo que podríamos llamar su «contenido»-; más bien, lo hace por su pertenencia al sujeto que lo concibe, es decir, a su posesor. «Mi idea [de pirámide] no es la idea de un objeto individual, pero es una idea que pertenece a un sujeto individual (...) Está individuada por su presencia en mi mente más que en la de otro» (Kenny 1993, 141). En su opinión, la solución de Tomás de Aquino para resolver la inmaterialidad del intelecto no está bien encaminada. Por así decirlo, no se puede esperar que el contenido de los conceptos resuelva el problema. De ahí la importancia que tiene para él la individuación de los objetos pensados. Esa individuación remite la solución a la cuestión planteada - si las formas puras prueban la inmaterialidad de la mente-al problema mente-cerebro. Y como Kenny se ha planteado ese problema en otro lugar, la prueba de Tomás de Aquino, que según Kenny está basada en una confusión, no resuelve la pregunta por la inmaterialidad del intelecto (Kenny 1993, 143).

A pesar de eso, podríamos preguntarnos: ¿qué ha demostrado Kenny con este análisis? Reconducir el problema de las formas puras al de la individuación de lo pensado intenta simplemente detener el razonamiento en sus premisas, eludiendo su punto central. El argumento de Tomás de Aquino se basa en la existencia de una capacidad de captar universales, es decir, de conceptos que no son equiparables a las propiedades individuadas de la materia. Al sostener que los conceptos no nos dicen nada que permita extraer conclusiones sobre su comprensión, el argumento de Tomás de Aquino deja ya de ser planteable. Y la posibilidad que éste plantea es que sin la afinidad cualitativa entre las «formas puras» y el entendimiento, éste nunca podría llegar a captar universales. Por consiguiente, razona Haldane (Haldane 2010, 135-136) Kenny no ha logrado desarticular el argumento de las formas. Para cuestionar la validez de este argumento, debería cuestionarse la pertinencia de extraer conclusiones sobre la ontología de una capacidad o su operación a partir de la naturaleza de sus objetos intencio- 
nales, como han hecho filósofos como J. Novak (Novak 1987) o R. Pasnau (Pasnau 1998). Ambos afirman que el paso de la intencionalidad a la ontología no está justificado. ¿Están en lo cierto? Analizar sus puntos nos llevaría demasiado lejos. Lo que, con todo, parece evidente es que el argumento de Tomás de Aquino no ha perdido actualidad, y que sólo examinando cuidadosamente sus premisas se puede sopesar su validez filosófica.

\section{Pensamiento e intencionalidad}

El cap. 9, titulado «el entendimiento», trata de algunas propiedades de lo mental como la intencionalidad o la diferencia entre pensar (thinking) y creer (believe), aunque no en el sentido fiducial que el verbo «creer» tiene en español e inglés, sino más bien en el sentido que ambos verbos comparten como sinónimos.

Antes de entrar en lo que caracteriza a cada uno de estos verbos, conviene señalar que son verbos intencionales, es decir, expresan una propiedad que ha calificado como «intencionalidad». Encontrar una definición precisa de intencionalidad es bastante difícil. De modo general, se puede decir que «la intencionalidad es la relación entre el pensamiento y aquello sobre lo que versa» (Kenny 1989, 124). Para Kenny no merece la pena buscar esa definición analizando las propiedades semánticas de los llamados «verbos psicológicos» como «pensar», «creer», «desear» o «tener la intención de», pues es tal la variedad de situaciones y sentidos de esos verbos que sería ingenuo pensar que bastan una o dos generalizaciones para dar con la esencia de la intencionalidad del pensamiento (Kenny 1989, 125).

En lugar de proponer una definición, Kenny opta por analizar el uso de algunos verbos intencionales. Una exposición del modo en que se emplean puede decirnos más que una definición. En este sentido, lo primero será establecer la distinción entre lo intencional y lo no-intencional. Lo intencional se diferencia de lo no-intencional en que tiene o describe un objeto de características semánticas, no físicas. Compárese p. ej. el verbo «pensar» con el verbo «quemar». Si uno desea saber si su tostada se ha quemado, lo que tiene que hacer es sacarla de la tostadora y examinarla. En cambio, si 
desea saber en qué está pensando una persona, no sirve de nada examinar a la persona en cuestión. «Las acciones intencionales son acciones que no producen un cambio en su objeto» (Kenny 1989, 124). Es el sujeto el que cambia y se modifica al pensar, y no físicamente, a pesar de que para pensar sea necesario experimentar ciertos cambios físicos. Lo que cambia con el pensamiento son sus estados conscientes.

Otra característica de lo intencional es que los objetos intencionales no tienen por qué existir. La expresión «esta tarde lloverá» tiene un objeto distinto de la expresión «Kenny dice que esta tarde lloverá»; en el primero, el objeto al que se refiere es claro y está definido; en el segundo la referencia es oblicua, pues apunta por un lado a un sujeto, y por otro, a una opinión. De este modo, la identificación del referente concreto es menos evidente. Cuanto más compleja resulta la proposición, más difícil es identificar adecuadamente su referente. «Cuando una persona tiene el pensamiento de que $p$, el contenido de su pensamiento tiene la misma complejidad que la frase a la que daría expresión si llegara a expresarse» (Kenny 1989, 126).

Ejemplos como los anteriores nos muestran que «pensar» $\mathrm{y}$ «creer» son verbos intencionales de gran riqueza. Aunque a veces se puedan emplear indistintamente como sinónimos, uno y otro nos revelan aspectos distintos de la mente. A menudo empleamos «pensar que $p$ » en el sentido de «Juan está pensando que $p$ ». En este contexto, «pensar» apunta más bien a un estado, el estado mental de Juan. En cambio, «creer que $p$ » no tiene esa connotación; p. ej., no decimos que «Juan está creyendo que $p$ », sino «Juan cree que $p$ ». La creencia muestra así una disposición estable y duradera, mientras que pensar, en el sentido de «X piensa que $p$ » no muestra esa misma disposición, sino que pone de manifiesto un episodio (Kenny 1989, 125-126).

La sugerencia de que la gramática del verbo «pensar» lo asemeja a un episodio debe tratarse con gran cuidado. Muchos manuales de psicología al uso definen el pensamiento como un proceso: el proceso de pensamiento. Y la pregunta que se formula habitualmente es: ¿cómo es posible que un proceso llegue a tener significado? Así, la confusión parece introducirse ya en el modo de plantear la pregunta. «Estamos tentados de pensar (...) 
que significar y comprender son procesos que son simultáneos y acompañantes del hablar, escuchar y escribir» (Kenny 1989, 130). Pero es un error. Comprender y significar no son concebibles como procesos mentales en el sentido en que concebimos los procesos físicos. La explicación de porqué resulta bastante ilustrativa. «Para un momento mientras lees, levanta los ojos del papel y recita el alfabeto sin mover los labios ni hacer ningún ruido. Esto es algo que se podría describir como un proceso mental. Es mental en el sentido de que es algo 'hecho en la cabeza'; nadie lo puede percibir, a diferencia de haber recitado el alfabeto en voz alta. Sólo preguntándote podría alguien que entra en la habitación saber lo que estabas haciendo» (Kenny 1989, 130-131). Recitar el alfabeto mentalmente puede definirse como un proceso mental que tiene comienzo, principio y fin. El proceso puede interrumpirse; el teléfono podría sonar mientras lo recitamos o incluso podríamos atascarnos y perdernos en medio de este proceso.

Sin embargo, comprender y significar no son procesos de esta índole. «Si significar fuese un proceso mental como éste, sería posible significar una oración sin que la oración tenga que proferirse. ¿Pero cómo significa una oración que no se dice?» (Kenny 1989, 131). El único modo de hacerlo sería recitarla para uno mismo o mentalmente, pero al hacerlo se construye una oración con sentido. Así, el comprender y el significar no son fenómenos que acompañan concomitantemente a la significación de las palabras, extendiéndose por un tiempo y desapareciendo cuando acaban de significar. «La comprensión no puede pensarse como un proceso en absoluto. Comprender es una habilidad, y por tanto es un estado más que un proceso (...) Pero es importante advertir de una posible malinterpretación. Comprender puede ser un estado, pero no un estado psicológico similar al dolor, la depresión o la excitación anímica. Estos estados duran, pueden continuarse o interrumpirse. Pero uno no puede saber ininterrumpidamente lo que significa una palabra» (Kenny 1989, 131).

La afirmación de que comprender y significar no son procesos mentales está orientada a respaldar y comprender mejor su visión de la mente como una capacidad de capacidades. Pensar es una capacidad central de la que dependen otras que están vinculadas al pensamiento como comprender 
y significar. Si esto es así, la gramática de los verbos «pensar», «comprender» $\mathrm{y}$ «significar» contrasta con la gramática de los procesos físicos como «quemar», «arrastrar» $\mathrm{y}$ «recoger», y de sus correspondientes usos verbales. Es evidente que los verbos «quemar», «arrastrar» $\mathrm{y}$ «recoger» expresan la ejecución de procesos de carácter físico, y no puramente semántico, con un uso lingüístico que es cualitativamente distinto. A juicio del autor, este contraste manifiesta un rasgo fundamental en la naturaleza de los actos intencionales, y es que no están sometidos a las leyes físicas y causales. La cita Wittgenstein en el apartado sobre la relación mente-cerebro ya lo ha puesto de manifiesto.

\section{Determinismo y libertad}

El último capítulo trata aspectos relacionados con el carácter científico de la psicología. Ryle dedicó el último capítulo de su obra al estatuto de la psicología como ciencia. En el Prólogo, Kenny señala que en este capítulo «no se propone dar un tratamiento sistemático o una evaluación de la investigación empírica reciente sobre la naturaleza de la mente. Más bien hay, en cada caso, un intento de mostrar en qué sentido la filosofía puede proveer un fundamento o encontrar los límites del estudio empírico de la mente» (Kenny 1989, viii).

En efecto, parte de los objetivos de Kenny en este capítulo está en senalar los límites de la comprensión científica del cerebro y los fenómenos de la mente. El prestigio de que goza la ciencia experimental le granjea credibilidad. Por eso, la filosofía ha adoptado a veces esquemas metodológicos de carácter científico, o lo que es lo mismo, esquemas cientistas. Esta adopción es frecuente en algunos círculos neoempiristas de la filosofía analítica. Para algunos de estos autores, la filosofía debería convertirse en una ciencia especializada en el sentido en que lo son otras disciplinas científicas como la física o la microbiología; Kenny cuestiona implícitamente esas ideas.

Este capítulo aborda el determinismo en la filosofía de la mente, la polémica entre compatibilistas e incompatibilistas en el debate sobre la 
libertad, y la relación de la teoría de la selección natural con el lenguaje. Comentaremos fundamentalmente las dos primeras.

El determinismo es una teoría filosófica antigua. Según Kenny, el determinismo del que va a tratar se ha expresado en ocasiones como la visión para la que todo evento tiene una causa (Kenny 1989, 140). Si este determinismo es verdadero, será verdad que para cada evento E habrá siempre una causa anterior $\mathrm{C}$ de tal forma que, si se da $\mathrm{C}$, entonces se dará $\mathrm{E}$ y la aparición de E estará regida por alguna ley. Seguramente, en esta descripción podrían englobarse las más diversas clases de determinismos que puedan plantearse. Entre los determinismos más difundidos en ámbitos científicos, este capítulo examina los determinismos psicológicos, económicos o sociales (Kenny 1989, 142).

Cada clase de determinismo tiene su propia particularidad. El determinismo psicológico, p. ej., observa una relación de causa y efecto entre estados psicológicos y estados neurofisiológicos de un individuo, es decir, entre deseos y creencias, de un lado, y los movimientos corporales que se siguen de ellas, de otro. Kenny recuerda que, tal y como ha señalado ya (Kenny 1989, 131), deseos y creencias no son eventos ni procesos mentales que tienen lugar en nuestro interior. Por consiguiente, el determinismo psicológico se cae por su propio peso, puesto que estrictamente hablando no hay nada -ningún resorte o posible fuerza oculta - en nuestro interior capaz de causar esos movimientos. «Una acción voluntaria puede ser voluntaria sin que haya ningún evento mental, deseo o elección distinta de la acción misma que la precede o acompaña» (Kenny 1989, 144). Lo cual acarrea consecuencias no sólo para este género de determinismo, sino para otras formas posibles, ya que «toda forma de determinismo es incoherente por malinterpretar la naturaleza de los fenómenos mentales a los que apelan tácitamente en su formulación» (Kenny 1989, 145).

Pero así como el autor tiene una posición bien definida, y evidentemente contraria, al determinismo, su posición es menos comprometida en el debate entre compatibilistas e incompatibilistas. En términos sucintos, en este debate unos sostienen que la libertad es posible y otros que debe convivir, si existe, con el hecho insoslayable del determinismo. Los compa- 
tibilistas defienden que no hay problema en mantener que todos nuestros actos están determinados, a la vez que admiten que nuestros actos son el resultado de elecciones. Los deterministas dicen que hay libertad de espontaneidad, o sea, una capacidad de obrar por querer, pero no libertad de indiferencia, esto es, la clase de libertad que nos deja escoger entre alternativas. «Esta clase de compatibilismo», afirma Kenny, «es, en mi opinión, errónea» (Kenny 1989, 147), pues no se puede forzar la separación de la libertad de espontaneidad y de indiferencia; para que haya verdadera libertad necesariamente tienen que darse las dos.

Los incompatibilistas aciertan en señalar que no hay genuina libertad mientras no se reconozca la capacidad humana de escoger entre alternativas. «Pero no está claro que los incompatibilistas tengan razón al pensar que toda forma de determinismo debe descartar el poder obrar de otra forma. Puede que lo piensen así por haber analizado incorrectamente el concepto de 'poder'» (Kenny 1989, 147-148), o sea, poder obrar siempre de otra forma.

Hay un tipo de determinismo, a saber, el determinismo fisiológico, que puede convivir pacíficamente con la libertad de indiferencia. Según el determinismo fisiológico, la actividad humana está determinada por estados neurofisiológicos del cerebro y el sistema nervioso central (Kenny 1989, 146). El querer -en el supuesto de que haya un estado fisiológico o cerebral que se corresponda con el querer- estará conectado con nuestras acciones de la forma que lo prevean las pertinentes leyes causales. Según esas leyes, habrá un estado fisiológico E1 que se corresponda con un curso de acción posible, y otro estado, E2, que se corresponda con otro distinto. La elección de una alternativa será contemporánea a la ocurrencia de E1 o E2, pero no de ambas a la vez o siempre de la misma. Kenny descarta que los estados puedan ser unidireccionales, de forma que el estado anterior a E1 o E2 -llámesele a este estado previo «E0»- entrañe necesariamente la ocurrencia de E1 (que no es, repitámoslo, el querer, sino el estado fisiológico que lo acompaña). De nuevo, sin importar el estado en el que un sujeto se encuentre al tomar una decisión, el determinismo fisiológico preserva la libertad de indiferencia (Kenny 1989, 149). 
Kenny asegura así que el compatibilismo es conciliable con un determinismo de bajo perfil (soft determinism), mientras que sería irreconciliable con el más ambicioso (hard determinism). Para evitar posibles malinterpretaciones sobre el alcance del determinismo fisiológico, el autor señala que esta visión no tiene porqué comportar la aceptación de un determinismo psicológico - el cual, ya no sería un determinismo de lo físico sino de lo mental-, porque no hay, ni tiene por qué haber una ley que correlacione los estados fisiológicos y psicológicos. El autor no ve nada que nos induzca a creer que los estados del cuerpo, o del cerebro, provocan causalmente, por ley, estados psicológicos (Kenny 1989, 149).

A pesar de aceptar el determinismo fisiológico como posibilidad, Kenny se declara agnóstico con respecto al éxito teórico del programa determinista en general. «No sé de ninguna razón convincente por la que creer que el determinismo universal es verdadero, y ninguna razón por la que pueda ser falso. No sé incluso si se le puede dar una formulación del todo coherente. La mayor parte de la gente que tiene una fe inconmovible en el determinismo o el indeterminismo, a mi entender basa su convicción en un acto de fe, o como mucho, en una extrapolación de la historia de la ciencia» (Kenny 1989, 150). Según Kenny, la ciencia no resuelve de suyo el problema del determinismo, de modo que es ambivalente; según sea el periodo de la ciencia que se mire se encontrará justificación para una tendencia u otra.

Naturalmente, Kenny reconoce que nuestro instinto filosófico, al menos el instinto de un lector que haya llegado a este punto, será probablemente indeterminista. Este instinto le llevará a defender la libertad. Sin embargo, si, como ha pretendido mostrar, la libertad es compatible con un determinismo de bajo perfil, su interesada defensa no tiene porqué legitimar el indeterminismo (Kenny 1989, 150). La cuestión está planteada y debe resolverse en el debate acerca de la naturaleza de las leyes que gobiernan el universo. Si esas leyes gobiernan los acontecimientos de modo tal que la naturaleza obra por necesidad en lo que a esas leyes se refiere, el filósofo no puede por más que aceptar los términos que se dan.

Sin embargo, si se pierde la visión de conjunto se corre el peligro de malinterpretar la postura del autor. Kenny advierte que la libertad que ha 
defendido - la de indiferencia- es característica de hombres y animales. Recuérdese que un animal superior puede elegir genuinamente entre las alternativas que se le presentan. Y se pregunta: ¿tiene la libertad individual humana algo que excluya el determinismo o el indeterminismo? Por desgracia, el autor no responde a esta cuestión. Con todo, los capítulos anteriores contienen elementos suficientes para responderla. Y es que lo característico de la voluntariedad humana es su intencionalidad. «Las acciones intencionales son aquellas que se escogen como fines en sí mismas o como medios para otros fines; si las acciones se escogen por sí mismas ya no son sólo intencionales sino que pueden ser llamadas propósitos» (Kenny 1989, 45). En el capítulo sobre la voluntad se recordó que para obrar por razones u obrar intencionalmente, es preciso ser capaz de dar esas razones y manifestar propósitos. Para mostrar que se posee esta capacidad se requiere ser capaz de dar razones, no simplemente conocerlas (Kenny 1989, 39).

Por eso, aunque Kenny no resuelve la cuestión de si hay algo en la libertad humana que excluya el determinismo o el indeterminismo, podría articularse una respuesta tentativa siguiendo lo que ha mantenido en otros lugares (Kenny 1973). De ahí se sigue que la acción humana puede ser llamada libre sin ambages con un determinismo de bajo perfil. Los elementos sobre los que podría basarse esta defensa de la libertad humana son:

a) El hecho de que el determinismo fisiológico concierne a las relaciones causales entre estados del cerebro y los movimientos corporales; en ningún caso a los estados psicológicos.

b) La voluntariedad que parece comprometida en el determinismo es la común a hombres y animales. Es la llamada libertad de indiferencia. Pero podría añadirse algo más. En realidad, si este determinismo afecta únicamente a estados fisiológicos, la teoría se compromete poco con el determinismo que impide la libertad, pues según lo anterior los estados psicológicos no son afectados por él.

Por último, no está claro en la perspectiva de Kenny, que los estados fisiológicos sean suficientes para establecer el contenido de una acción específicamente humana, ya que esto viene generalmente definido por la 


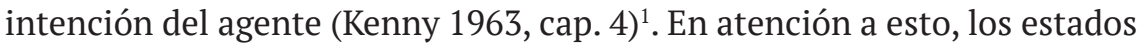
fisiológicos no son condición suficiente para garantizar que un agente humano obra libremente. Para explicar el obrar libre sería preciso ir más allá, o sea, conocer las intenciones del agente; sin ese conocimiento sólo se podría describir la conducta humana desde un punto de vista meramente físico, lo que haría a esa «descripción» incompleta.

Lo lógico, pues, sería concluir que la libertad cae más allá del área de influencia tanto del determinismo como del indeterminismo; estrictamente hablando, el determinismo afecta sólo a la dimensión material del obrar. Sólo el individuo completo, y no sólo su cuerpo o su cerebro de forma aislada, puede actuar según la específica voluntariedad humana. En ese sentido, Kenny deja de mencionar un hecho bastante relevante. Y es que el determinismo cubre con su explicación la dimensión física o material de la conducta, pero es ineficaz en lo que excede a esa dimensión. Se le escapa la dimensión formal o intencional de la conducta, que es justamente la más relevante, la que concierne a las intenciones y motivos de un agente.

En la medida en que esta crítica descansa sobre una omisión, quizá no sea la más eficaz. En cambio, habría mucho que decir sobre la definición de determinismo como tal. La concepción del universo que surge de la idea de que, dada una causa anterior $\mathrm{C}$, se seguirá un evento $\mathrm{E}$, y que esa conexión es explicable por ley parece cuestionable. Ciertamente, el autor no desea entrar en el debate de qué constituye un evento de estas características (Kenny 1989, 140-141), pero tal vez debería haberlo hecho si considera al determinismo de bajo perfil como un serio aspirante a explicar al menos parte de la conducta voluntaria. La principal debilidad que tiene la aceptación de un planteamiento determinista que no precisa qué son específicamente estos eventos no es difícil de imaginar; especialmente cuando se trata de dar cuenta del movimiento propio de organismos biológicos, que son la clase de sustancias más complejas. La razón es que la aparición de causas no está necesariamente vinculada a sus efectos, ni los efectos están, por así decirlo, predeterminados por su causa.

Lugar donde el autor explica cómo los motivos permiten dar cuenta de la intencionalidad de la acción humana. 
A este fenómeno se le ha conocido tradicionalmente como el azar. Aristóteles, que se ocupó de él en la Física, sabe que hay quienes piensan que «hay una causa determinada para cuantas cosas decimos que se originan como consecuencia de la fortuna o de la espontaneidad» (Aristóteles 1996, 196a 1-3), y por tanto, que nada viene a ser por azar sino que cada cosa está predeterminada a ser como es por naturaleza. Para el determinista, en el universo físico no existen novedades genuinas, ya que todos los movimientos de un móvil estarían limitados a un rango más o menos estrecho de posibilidades predecibles. Aristóteles rechaza esta idea porque entiende que la dificultad de comprender el azar no estriba en que carezca de causa, sino en que su causa es indeterminada. Considera que el azar aparece por la confluencia de causas que generan efectos contingentes, es decir, efectos que siendo de una determinada manera podrían ser perfectamente de otra. Como estos eventos no provienen de una causa per se o necesaria, sino per accidens, su predicción sería imposible además de irrelevante, porque la ciencia no puede estudiar causas per accidens. Por eso, señala que «es acertado decir que la fortuna es algo que escapa al raciocinio, porque el raciocinio se ocupa bien de las cosas que son siempre o de las que son por lo general, mientras que la fortuna está entre las cosas que se producen al margen de éstas» (Aristóteles 1996, 197a 18-21).

Si Aristóteles está en lo cierto, no parece claro, ni mucho menos, posible, que todo evento $\mathrm{E}$ provenga necesariamente de una causa $\mathrm{C}$, y que el vínculo entre E y C sea explicable por ley. El azar no es una ficción, sino que existe (Aristóteles 1996, 196a 11-15, 196b 15). El determinismo fisiológico acepta implícitamente que es posible conocer el origen de todos los efectos físicos, o dicho en otras palabras, que el azar no existe. Sin embargo, esta visión parece más bien el resultado de un esquema mecanicista en el que, por expresarlo así, todas las fuerzas que inciden sobre el móvil son de carácter externo y están determinadas ab initio, de forma que su posición inicial contiene la clave de todo. Esta postura no sospecha que los efectos de la causalidad son mucho más complejos. Esto se manifiesta especialmente en los organismos que dan lugar a novedades genuinas, que son los organismos vivos. Hoy sabemos que la capacidad de generar novedades de un sistema vivo no está simplemente 
en su $\mathrm{ADN}$, ni en su interacción con el medio, sino en algo más profundo. Y es que en los organismos los aspectos formales de la causalidad pesan más que los materiales. $\mathrm{Si}$, como se ha mencionado, el ADN se define por sus instrucciones más que por su composición, sus aspectos formales son los más importantes; y así, el organismo es más que su ADN. Por esto, es impropio esperar que, en un viviente, la aparición de una causa $C$ entrañe necesariamente y sin remisión la aparición de un evento E que puede y debe explicarse por ley. La causalidad, especialmente en el ámbito complejo de la biología, no es siempre predecible, por lo que el análisis mecánico del viviente es poco esclarecedor. Como se sabe, en la perspectiva aristotélica del viviente, la causa formal y final tienen mayor peso que la causa eficiente y material.

Lo señalado pone obstáculos al intento de establecer un determinismo de bajo perfil como el determinismo fisiológico. Por eso, resulta curioso que Kenny se sienta obligado a intervenir en este debate una vez que ha señalado que no ve razones que avalen la defensa de un determinismo universal, y que duda también de que el determinismo pueda tener una formulación del todo coherente (Kenny 1989, 150). Si no existen razones suficientes para dirimir el debate entre el determinismo o el indeterminismo, tampoco debería haberlas para aceptar el determinismo fisiológico, y por tanto, para considerar que el determinismo de bajo perfil es una seria amenaza para la libertad.

\section{Conclusión}

El principal mérito de la obra de Kenny consiste en haber articulado una original y valiosa síntesis entre el pensamiento de Wittgenstein y la tradición aristotélica. En años anteriores a la publicación de este libro, Kenny afirmó que «si Wittgenstein estuvo en lo cierto, la filosofía se habría descaminado desde tiempos de Descartes y debería rectificar el rumbo en una dirección más acorde con el pensamiento medieval» (Kenny 1980, 28). Aunque Wittgenstein ignoraba el pensamiento medieval tanto como el de Platón o Aristóteles, su particular percepción de los males de la filosofía moderna y contemporánea le llevó a pensar que la filosofía necesitaba rectificar se- 
riamente el rumbo para ajustarse a lo que debía ser, esto es, un saber que lograse disolver los problemas provocados por preconcepciones de la realidad ocultas en el lenguaje, que sólo salen a la luz a través de una singular terapia.

Más allá de la validez de este método, que Kenny sólo sigue parcialmente, la sugerencia de que la filosofía perdió el rumbo con Descartes es una luz especialmente valiosa en el contexto presente. Por tanto, el análisis de los prejuicios que la filosofía contemporánea ha heredado del cartesianismo sigue siendo actual. La filosofía de la mente contemporánea parece estancada en algunas premisas cartesianas, lo que se aprecia p. ej. en el planteamiento del problema de la conciencia en un mundo material. Algunos autores contemporáneos han llamado la atención sobre el sustrato cartesiano de muchas de las soluciones que se ofrecen actualmente al problema mente-cerebro. Burnyeat (Burnyeat 1992, 16) aseveró que mientras que la filosofía se ha centrado en el análisis las «propiedades conscientes», la concepción cartesiana de la materia sigue en pie, de tal forma que el problema mente-cuerpo, tal como está planteado, ha hecho incomprensible la física de Aristóteles, lo que a su vez hace difícil de comprender su solución al problema mente-cerebro. De esta forma, la concepción cartesiana del mundo, y especialmente de la materia, introduce importantes obstáculos en el progreso de la filosofía de la mente.

La tradición medieval, y especialmente el pensamiento de Tomás de Aquino en la cuestión sobre el hombre de la Summa Theologiae, están fuera de la órbita cartesiana. Su peculiar visión del ser humano como una sustancia hilemórfica viva que aúna en sí la capacidad de razonar y ser libre, así como su necesidad de sostener el pensamiento sobre facultades orgánicas, hace su visión especialmente actual. En una etapa en la que el cientismo, el neodarwinismo, las diversas formas de fisicalismo y eliminativismo se han convertido en las posturas más representativas del pensamiento analítico contemporáneo, sorprende el gran valor de algunos conceptos de la tradición aristotélica que eluden algunos problemas persistentes. En este sentido, la vuelta a esta tradición de The Metaphysics of Mind representa una indudable aportación, y señala el camino a seguir para quienes desean hacer progresar la discusión más allá del actual marco de coordenadas. 


\section{Referencias}

Aristóteles. 1978. Acerca del alma, translated by J. L. Calvo. Madrid: Gredos.

-. 1996. Física, translated by J. L. Calvo. Madrid: CSIC.

-. 2000a. Ética Nicomaquea, translated by J. Pallí. Madrid: Gredos.

-. 2000b. Política. Madrid: Gredos.

Burnyeat, M. 1992. "Is an Aristotelian Philosophy of Mind Still Credible? (A Draft)".

In Essays in Aristotle's De Anima, edited by M. Nussbaum, and A. O. Rorty, 15-26, Oxford: Oxford University Press.

Crane, T. 2000. “The origins of qualia.” In History of the Mind-Body Problem, edited by T. Crane, and S. Patterson, 169-194. London: Routledge.

Davidson, D. 1980. Essays on Actions and Events. Oxford: Clarendon Press.

Glock, H.-J. 2009. “Can Animals Act For Reasons?” Inquiry 52 (3): 232-254.

Hacker, P.M.S., and J. Cottingham. 2010. Mind, Method, and Morality: Essays in Honour of Anthony Kenny. Oxford: Oxford University Press.

Haldane, J. 2010. "Kenny and Aquinas on the Metaphysics of Mind.” In Mind, Method, and Morality: Essays in Honour of Anthony Kenny, edited by P.M.S. Hacker, and J. Cottingham, 119-139. Oxford: Oxford University Press.

Kenny, A. 1963. Action, Emotion and Will. London: Kegan Paul.

-. 1973. "Freedom, Spontaneity and Indifference.” In Essays on Freedom and Action, edited by T. Honderich, 87-104. London: Routledge and Kegan Paul.

-. 1980. Aquinas. Oxford: Oxford University Press.

-. 1989. The Metaphysics of Mind. Oxford: Clarendon Press.

-. 1993. Aquinas on Mind. Oxford: Oxford University Press.

-. 2008. “Cognitive Scientism.” In From Empedocles to Wittgenstein: Historical Essays in Philosophy, edited by A. Kenny, 149-162. Oxford: Clarendon Press.

MacIntyre, A. 1999. Dependent Rational Animals. Why Human Beings Need the Virtues. Illinois: Open Court.

Novak, J. 1987. “Aquinas and the Incorruptibility of the Soul.” History of Philosophy Quarterly 4 (4): 405-421.

Pasnau, R. 1998. “Aquinas and the Content Fallacy.” Modern Schoolman 75: 293-314. Ryle, G. 1949. The Concept of Mind. London: Hutchinson.

Tomás de Aquino. 1950-1956. Summa Theologiae, edited by P. Caramello. Taurini: Marietti.

Wittgenstein, L. 1953. Philosophical Investigations, translated by G.E.M. Anscombe. Oxford: Blackwell.

-. 1974. Zettel. Oxford: Blackwell. 